\title{
Buenos docentes universitarios: ¿Qué dicen los estudiantes?'
}

\author{
Eugenio Merellano-Navarro" \\ Alejandro Almonacid-Fierro" \\ Alberto Moreno-Doñall! \\ Cesar Castro-Jaque"
}

I- Trabajo financiado por el fondo de Investigación Interno de la Universidad Autónoma de Chile, código DPI26-2015, titulado: Caracterización de un buen docente universitario según la percepción de estudiantes, docentes y directores de carrera de la Facultad de Educación de la Universidad Autónoma de Chile-Talca.

II- Universidad Autónoma de Chile, Talca, Chile.

Contactos: emerellanon@uautonoma.cl aalmonacidf@uautonoma.cl ccastroj@uautonoma.cl

III- Universidad de Valparaíso, Valparaíso, Chile.

Contacto: alberto.moreno@uv.cl

\section{Resumen}

Los procesos de transformación histórica que está viviendo la educación son el resultado de las necesidades sociales que están impulsando los cambios y la reformulación de los sistemas establecidos. La docencia universitaria debe asumir, desde esta perspectiva, nuevas responsabilidades que trasciendan el rol del docente en procura de mejorar los procesos de enseñanzaaprendizaje de manera eficiente y con calidad. El objetivo de la investigación es conocer las características de un buen docente universitario según la percepción de los estudiantes. Se realizó a partir de un estudio de caso, con estudiantes de la Facultad de Educación de la Universidad Autónoma de Chile, sede Talca. La metodología utilizada es mixta, con instrumentos cualitativos y cuantitativos que permitieron recoger la información a través de cuestionarios de preguntas abiertas y entrevistas en profundidad, que dan cuenta de los resultados con perspectivas complementarias que identifican las características más relevantes según la percepción de los estudiantes del desempeño del buen docente universitario. A través de un análisis de frecuencia, en la fase cuantitativa, y otro de contenido de los discursos en la fase cualitativa, los estudiantes identifican las características más importantes a la hora de valorar a un buen docente universitario. Los resultados más destacados señalan que los rasgos interpersonales son la categoría con mayor frecuencia junto al dominio del contenido de la enseñanza. Se concluye que los estudiantes valoran a un buen docente cuando posee características que se conjugan en lo pedagógico, humano e ideológico, priorizando en todo momento, la centralidad en el aprendizaje de los estudiantes.

\section{Palabras clave}

Docentes universitarios - Calidad docente - Efectividad docente Evaluación - Calidad. 


\title{
Good university teachers: what do students say about them?
}

\author{
Eugenio Merellano-Navarro" \\ Alejandro Almonacid-Fierro" \\ Alberto Moreno-Doñall! \\ Cesar Castro-Jaque"
}

I- Work funded by the internal Fund of research of the University Autónoma of Chile, code DPI26-2015, entitled: characterization of a good university faculty member according to the perceptions of students, teachers and directors of the Faculty of Education of the University Autónoma of Chile, Talca.

II- Universidad Autónoma de Chile, Talca, Chile.

Contacts: emerellanon@uautonoma.cl aalmonacidf@uautonoma.cl ccastroj@uautonoma.cl

III- Universidad de Valparaíso, Valparaíso, Chile. Contact: alberto.moreno@uv.cl

\section{Abstract}

The historical processes of transformation that the education system is experiencing are the result of the social needs that are driving the changes and the reformulation of established systems. From this perspective, teaching practices at the University level must consider the new responsibilities that transcend the role of the teacher, in order to improve the processes of teaching and learning in an efficient manner and with quality. The objective of this research is to identify the characteristics of a good University faculty member according to the perception of the students. This research was based on a case study with students from the Faculty of education at Autónoma University of Chile, Talca headquarters. The methodology used in this study is mixed, with qualitative and quantitative instruments that helped to collect data through questionnaires of open questions and interviews in depth, giving account of the results with complementary perspectives that identify the most relevant characteristics, according to the students' perceptions, of the performance of good university teachers. Through an analysis of frequency, in the quantitative phase, and another one about the content of the speeches in the qualitative phase, the student's identified the most important aspects when evaluating a good faculty member. The most outstanding results indicate that the interpersonal aspect of the teacher is the most frequent category, along with the domain of the content of teaching. It is concluded that the students value a good teacher when he has features that are combined in the pedagogical, human, and ideological aspects and, those teachers that prioritize the learning process of the students at all times.

\section{Keywords}

University professors - Teaching quality - Teaching effectiveness Evaluation - Quality. 


\section{Introducción}

Los avances en el ámbito educativo, específicamente el cómo enseñar y cómo se aprende, han potenciado el desarrollo de requerimientos de calidad del sistema educativo, los que apuntan principalmente a los actores que participan en los procesos formativos. En el ámbito concreto de la educación superior, la docencia universitaria ha sido foco de fuertes críticas que cuestionan la labor de los docentes (CABALÍn et al., 2010). Esto ha llevado a numerosos intentos por transformar el trabajo pedagógico de dichos profesionales (CAREAGA, 2007; LÓPEZ et al., 2010), asumiendo nuevas responsabilidades y desafíos que los han conducido desde un quehacer orientado, casi exclusivamente, desde el traspaso de información, a un docente que oriente su hacer pedagógico hacia el desarrollo de procesos formativos orientado a la construcción de diversas competencias, cuyo propósito es el desempeño exitoso, comprometido y creativo en el ámbito profesional (BOTELLO; BONVALLET, 2002; CORRÊA; RIBEIRO, 2013; LOPÉZ DE MATURANA, 2012).

Desde estos cambios, la labor del docente universitario exige, hoy, una disposición a conocer y comprender a los alumnos en sus particularidades individuales y situaciones, así como la evolución de los mismos en el contexto de aula (SHULMAN, 1987; 1986; TARDIF, 2004). Una docencia de calidad en el ámbito universitario, entonces, implica que el docente esté constantemente reflexionando y reconstruyendo sus estrategias de enseñanza, alejándose de un rol ligado a la transmisión acrítica de conocimientos y acercándose a un trabajo orientado hacia la guía y generación de ambientes formativos donde los alumnos sean capaces de crear y construir sus propios aprendizajes, diversificando las estrategias metodológicas e individualizando la docencia en función de las particularidades de sus estudiantes (MUNDINA; POMBO; RUIZ, 2005). Debido a la labor del docente y a la importancia de velar por un proceso significativo en la formación, es trascendental conocer la relevancia de los actores que influyen en los procesos de educativos. Delors (1996) y Freire (1978) señalan que el proceso de enseñanzaaprendizaje no debe ser unidireccional, sino más bien un proceso de interrelación entre el profesor y el estudiante, favoreciendo así los cuatro pilares de la educación: aprender a conocer, aprender a hacer, aprender a convivir y aprender a ser. Al considerar lo indicado, la labor docente es un compromiso de por vida, lo que involucra una serie de conductas, actitudes sociales, valóricas y emocionales, que se deben tener en cuenta día a día al momento de ejercer la pedagogía, debiendo ser el principal objetivo de cada uno de los profesores: ir más allá de la entrega conocimientos y contenidos, que el estudiante pueda trascender al desarrollo personal y social.

En este contexto de cambio, diversos autores han abordado y estudiado las principales características y/o competencias que debe poseer un profesor para ser calificado como un buen docente en el contexto universitario (CASERO, 2010; FERNÁNDEZ; GONZÁLEZ, 2012; GARCÍA; DOMINGO; DÍAZ, 2006; LUNASERRANO; VALLLE-ESPINOSA; OSUNALEVER, 2010; RICE, 2003).

Cabe destacar la clasificación que realizan Ventura et al. (2011) sobre las características de los buenos docentes distinguiéndose las siguientes:

a) El docente debe cumplir con una seria de características pedagógicas relacionadas con la actualización docente, su implicación en procesos investigativos sobre su propia enseñanza y una constante reflexión pedagógica sobre su quehacer profesional.

b) Además, debe mostrar dominio conceptual y teórico sobre el contenido propio de su enseñanza; capacidad para usar diferentes metodologías en función de las características de sus estudiantes; y poseer una actitud pedagógica que invite al aprendizaje; explicar de manera contextualizada. 
c) Por último, debe ser consciente de una serie de características que transcienden su propio ámbito disciplinar y que están relacionadas con la capacidad de acompañar a sus estudiantes en las inquietudes, dificultades y expectativas que estos tengan.

Es interesante, en este contexto, mostrar la caracterización que realiza Calvo (2002) sobre lo que significa un buen docente universitario. Este buen docente debe entender y llevar a cabo un trabajo en el que las labores de docencia e investigación no son dos realidades dicotómicas, sino que forman partes inseparables de un quehacer pedagógico exitoso. Esta perspectiva está caracterizada por:

a) El misterio es el eje vertebrador del quehacer del profesor universitario: quien enseña asombra con el misterio, quien investiga lo hace asombrado por el misterio.

b) La invitación a un aprendizaje que permita construir y comprender regularidades: quien enseña muestra regularidades, quien investiga indaga sobre ellas.

c) Apertura inocente, no ingenua, ante sí y el mundo: la inocencia permite entender que el saber no es definitivo y que la ignorancia no es un lastre.

d) No tienen miedo al error, pues este es constitutivo del aprendizaje.

e) Entiende el conocimiento desde su paradoja: cada aprendizaje genera nuevas preguntas que permiten seguir conociendo.

Por último, señalamos la caracterización del buen docente universitario según Santos Guerra (1998):

a) Conocedor de su disciplina.

b) Abierto a la investigación y a la actualización del conocimiento.

c) Comprende qué es lo que sucede en el aula en la que posteriormente se desarrollarán los profesionales.

d) Sabe cómo aprenden los alumnos.

e) Conoce cómo se pueden organizar de mejor manera los espacios y tiempos pedagógicos.
El profesor, independiente del nivel educativo en el que enseñe, no es sólo un experto conocedor de una disciplina sino un especialista en el diseño, desarrollo, análisis y evaluación de su propia práctica con el objetivo central de alcanzar el mayor logro de aprendizaje en sus estudiantes (BOYD et al., 2009; HARRIS; SASS, 2011; SCHÖN, 2010).

En Chile, es responsabilidad del gobierno y del Ministerio de Educación velar porque las instituciones formadoras de profesores cumplan con las exigencias establecidas en los procesos formativos. Desde el año 2006, Chile cuenta con un Sistema de Aseguramiento de la Calidad -Ley $n^{\circ} 20.129$ (CHILE, 2006)-, que establece que el organismo encargado de verificar y promover la calidad de las Universidades, Institutos profesionales y centros de formación técnica, y de las carreras que estas instituciones ofrecen, es la Comisión Nacional de Acreditación (CNA). En el caso de las carreras de Medicina y Pedagogía la acreditación es obligatoria, según lo establece la Ley 20.129. Son los propósitos declarados por la institución, los estándares nacionales e internacionales y el proyecto de desarrollo académico de la institución los que la CNA, debe revisar e informar de sus resultados (CHILE, 2016). El sistema educacional y sus diferentes actores están siendo sometidos a permanentes evaluaciones que pretenden mejorar la calidad de la educación impartida por los profesores, el Sistema de Evaluación del Desempeño Profesional Docente y la Prueba Inicia, ambas evaluaciones pretenden dar cuenta del desempeño y del nivel de conocimientos que tienen los profesores. Las instituciones privadas poseen sus propias evaluaciones útiles para tomar decisiones administrativas y académicas con la finalidad de entregar una mejor educación.

En este contexto, la Universidad Autónoma de Chile, cuenta con un sistema de evaluación que tiene como objetivo revisar los procesos académicos desarrollados en la formación de estudiantes de pregrado. Cabe señalar que la evaluación es de docentes, estudiantes y directivos, siguiendo la modalidad 
de responder una encuesta finalizando cada semestre. Los docentes son evaluados por los Directores de Carrera y los estudiantes, a su vez deben responder una autoevaluación. Estas evaluaciones constan de una serie de criterios establecidos, de los cuales se deben evaluar con puntuación de uno a siete (escala de Likert), dependiendo del grado de cumplimiento en cada criterio. Las evaluaciones reflejan que cada criterio da cuenta de las principales características que debe poseer un buen docente para la institución. Es por ello, que es relevante estudiar el concepto de buen docente considerando a los diferentes actores involucrados en el sistema. La encuesta la responden los estudiantes, docentes y directores de carrera; los criterios de evaluación son entregados por la institución y no formulados por los principales actores del proceso formativo universitario, razón fundamental para realizar una investigación que aporte a la toma de decisiones de la universidad. El objetivo de este estudio es conocer las características un buen docente universitario según la opinión de los estudiantes de la Facultad de Educación de la Universidad Autónoma de Chile, sede Talca.

\section{Metodología}

El estudio se sustenta desde el diseño mixto al utilizar técnicas e instrumentos del ámbito cualitativo y cuantitativo. La aplicación y análisis de los instrumentos, marca la metodología utilizada. Los enfoques cualitativos y cuantitativos pueden ser perspectivas complementarias, toda vez que es posible utilizar las fortalezas de ambas lógicas investigativas, por otro lado se triangularán los datos recogidos a través de diferentes técnicas tanto cuantitativas como cualitativas, lo que permitirá aumentar la validez del análisis y las inferencias (DAVILA, 1999; MURCIA; JARAMILLO, 2000).

En un primer momento, se utilizará metodología cuantitativa al buscar la generalización de los comportamientos, acciones y experiencias de una población determinada (BISQUERRA, 1989), en este caso la Facultad de Educación. A través de esta metodología se pretendió abarcar la mayor cantidad de sujetos investigados de forma rápida y eficiente, permitiendo jerarquizar a partir de la frecuencia obtenida, las características que los estudiantes de la Facultad otorgan al buen docente universitario. Esta metodología permitió identificar las características más representativas que son consideradas por los estudiantes de $7^{\circ}$ semestre de dicha facultad.

Posteriormente, al tener esta primera aproximación de los resultados, se procederá a través del paradigma interpretativo y una metodología cualitativa, intentar comprender y describir un fenómeno único e irrepetible (RICOY, 2006). Además, se busca conocer la realidad desde los significados de las personas involucradas en la acción educativa, específicamente, sus percepciones acerca de un buen docente, características no observables directamente a través de la experimentación. A través de la interpretación de la subjetividad de los sujetos, se recoge la información con el fin de ayudar a los propios sujetos y al propio investigador en sus experiencias de aprendizaje. Esta etapa se llevó a cabo por medio de un instrumento cualitativo que fue aplicado a los estudiantes.

Además, se utilizó el estudio de caso, que según Simons tiene como propósito "investigar la particularidad, la unidad, del caso singular" (2011, p. 20). El caso es la Facultad de Educación de la Universidad Autónoma de Chile, sede Talca, Chile, en la que se imparten las carreras de Pedagogía en las siguientes especialidades: Artes Visuales, Matemática, Inglés, Lengua Castellana y Comunicación, Educación Física, Educación General Básica, Historia Geografía y Ciencias Sociales y Educación Parvularia.

Los instrumentos de recolección de información utilizados:

- Cuestionario de preguntas abiertas. Este instrumento posee tres preguntas abiertas que ya fueron usadas en investigaciones anteriores por Casero (2010). Se aplicó al 80\% 
de la población que cursa al $7^{\circ}$ semestre de un de las ocho carreras de Pedagogía pertenecientes a la Facultad de Educación de la Universidad Autónoma de Chile, sede Talca. El propósito del uso del cuestionario fue abarcar el mayor número de la muestra de una forma rápida y eficaz.

- Entrevistas semiestructuradas en profundidad. Tras la aplicación del cuestionario se eligieron a dos informantes clave de cada carrera para profundizar en las percepciones del estudiante sobre lo que significa ser un buen docente universitario.

La selección de los sujetos investigados se realizó bajo los siguientes criterios:
- Estudiantes matriculados en 40 año de Pedagogía.

- Asistencia el día de aplicación del cuestionario.

- Interés en participar en el estudio.

En la Tabla 1 se aprecian las carreras de la Facultad de Educación de la Universidad Autónoma de Chile, Sede Talca, y el total de estudiantes de $7^{\circ}$ semestre. También se distingue el número de estudiantes encuestados por carrera y los de sujetos clave, pertenecientes a las ocho carreras de la Facultad de Educación.

Tabla 1. Total de encuestas y entrevistas

\begin{tabular}{|c|c|c|c|}
\hline CARRERAS FACULTAD DE EDUCACIÓN & Total de alumnos & Cuestionarios aplicados & Entrevista \\
\hline Pedagogía en Educación General Básica & 47 & 34 & 2 \\
\hline Pedagogía en Educación Física & 90 & 78 & 2 \\
\hline Pedagogía en Educación Parvularia & 60 & 55 & 2 \\
\hline Pedagogía en Inglés & 65 & 60 & 2 \\
\hline Pedagogía en Historia Geografía y Ciencias Sociales & 45 & 37 & 2 \\
\hline Pedagogía en Lengua Castellana y Comunicación & 50 & 28 & 2 \\
\hline Pedagogía en Matemáticas & 18 & 16 & 2 \\
\hline Pedagogía en Artes Visuales & 26 & 11 & 2 \\
\hline TOTAL & 401 & 319 & 16 \\
\hline
\end{tabular}

Fuente: Elaboración propia.

Al ser un estudio de enfoque mixto, se siguió un procedimiento metodológico que utiliza al mismo tiempo palabras, número, textos y relatos de personas, que fueron analizados por medio de frecuencias y significados otorgados por los actores estudiados sobre las características del buen docente universitario.

Todos los datos obtenidos en la investigación, sean por el instrumento cuantitativo o cualitativo, se redujeron, codificaron y categorizaron por medio del software de investigación Nvivo 10. Se analizó el 100\% de los cuestionarios, agrupando los relatos en las tres categorías previas, surgidas de la revisión teórica: características humanas, características técnicas/ pedagógicas y características ideológicas. Por medio de la codificación y creación de nodos, se obtuvo un análisis de frecuencia que identificó las características más importantes por parte de los estudiantes de la facultad.

Para obtener la saturación de los datos, en una segunda etapa de obtención de información de los estudiantes, se realizaron dos entrevistas en profundidad por carrera. En este momento cualitativo, se redujo la información apoyado nuevamente por el programa Nvivo 10.

\section{Resultados}

Los cuestionarios aplicados abarcaron el 80\% de la totalidad de estudiantes del nivel $700^{1}$ de la Facultad de Educación. Además, se

1- Nivel 700 corresponde al séptimo semestre de formación. 
realizaron dieciséis entrevistas en profundidad (ver Tabla 1). A través de esta información se procedió a reducir datos obteniendo la siguiente matriz (ver
Tabla 2) que sintetiza las características del buen docente universitario según la percepción de los estudiantes de la Facultad de Educación.

Tabla 2- Matriz de sistematización categorías

\begin{tabular}{|c|c|c|c|c|c|}
\hline MEGA CATEGORÍA & CATEGORÍA PREVIA & CATEGORIA PRIMARIA & CATEGORÍAS SECUNDARIAS & $\%$ & Referencias \\
\hline \multirow{27}{*}{$\begin{array}{l}\text { Buen Docente } \\
\text { Universitario }\end{array}$} & \multirow{11}{*}{$\begin{array}{l}\text { Características } \\
\text { Pedagógicas }\end{array}$} & \multirow{4}{*}{ Perfil Pedagógico } & Dominio contenido de enseñanza & 23,3 & 463 \\
\hline & & & Continua capacitación & 2,7 & 55 \\
\hline & & & Contextualizado & 2,5 & 51 \\
\hline & & & Reflexivo & 1,4 & 28 \\
\hline & & \multirow{7}{*}{ Perfil Didáctico } & Calidad de la metodología de enseñanza & 10,2 & 203 \\
\hline & & & Metodología de enseñanza comprensible & 7,1 & 135 \\
\hline & & & Metodología de enseñanza didáctica & 6,3 & 125 \\
\hline & & & Proceso Evaluativo & 5,8 & 116 \\
\hline & & & Innovador & 1,3 & 25 \\
\hline & & & Dinámico & 2,6 & 52 \\
\hline & & & Creativo & 0,4 & 10 \\
\hline & \multirow{8}{*}{ Características Humanas } & \multirow{3}{*}{$\begin{array}{l}\text { Características } \\
\text { comunicativas }\end{array}$} & Expresivo corpóreos & 0.57 & 12 \\
\hline & & & Expresivo gráficos & 0.36 & 7 \\
\hline & & & Expresivo orales & 6.54 & 125 \\
\hline & & \multirow{3}{*}{ Características sociales } & Rasgos interpersonales & 34.78 & 582 \\
\hline & & & Rasgos actitudinales & 7.54 & 156 \\
\hline & & & Competente Idóneo & 5.6 & 112 \\
\hline & & \multirow{2}{*}{ Perfil profesional } & Docente Culto & 4 & 98 \\
\hline & & & Responsable & 16 & 302 \\
\hline & \multirow{8}{*}{$\begin{array}{l}\text { Características } \\
\text { Ideológicas }\end{array}$} & \multirow{2}{*}{$\begin{array}{l}\text { Características } \\
\text { Profesionales }\end{array}$} & Puntualidad-Compromiso & 6.4 & 136 \\
\hline & & & Ética laboral & 1.3 & 25 \\
\hline & & \multirow{3}{*}{ Valores profesionales } & Presentación personal & 1.6 & 33 \\
\hline & & & Vocación & 6.2 & 125 \\
\hline & & & Líder & 5.2 & 111 \\
\hline & & \multirow{3}{*}{$\begin{array}{l}\text { Características de } \\
\text { personalidad }\end{array}$} & Rasgos ético-morales & 11.77 & 286 \\
\hline & & & Rasgos humanístico-emocionales & 18.05 & 376 \\
\hline & & & Rasgos formativo-pedagógicos & 22.5 & 448 \\
\hline
\end{tabular}

Fuente: Elaboración propia.

En la Tabla 2, se presentan las categorías más significativas del estudio. Esta selección se realizó a través del porcentaje de frecuencia de la totalidad de las características que emergieron por categoría previa. El criterio de exclusión de esta tabla corresponde a todas aquellas características que presentaban una representación menor a 0,3\%. El número de referencia señala la cantidad de veces en que se mencionó en los relatos de los estudiantes de la facultad la característica descrita. Esta cifra está directamente relacionada con el 
número de porcentaje de relevancia de cada categoría secundaria.

Las características más predominantes en los estudiantes de la Facultad de Educación en la categoría previa Características Pedagógicas son: dominio de contenido y calidad de la metodología de enseñanza alcanzando entre ambas un 33,5\% de alcance en esta categoría. En relación a la categoría previa Características Humanas, los rasgos personales predominan con una representatividad de 34,78\% y sumada a la característica mencionada por los estudiantes responsabilidad alcanza un 50,78\% de la totalidad de representatividad en esta categoría. Por último, dentro de la categoría previa Características Ideológicas, los rasgos ético-morales, rasgos humanístico-emocionales y rasgos formativospedagógicos abarcan juntos un 52,32\% de impacto. Cabe destacar la importancia que atribuyen los estudiantes a las características humanas e ideológicas en contraste a las características pedagógicas, al poseer un mayor número de referencias en los recursos analizados.

A continuación, se presentarán los relatos más destacados de las categorías previas más representativas y su correspondiente discusión e interpretación.

\section{Categoría Previa Características Pedagógicas - Categoría Primaria Perfil Pedagógico - Categoría \\ Secundaria: Dominio del contenido de enseñanza}

Esta categoría posee un porcentaje de frecuencia de un 23,3 \% del total de los estudiantes investigados del nivel 700 de la Faculta de Educación de la Universidad Autónoma de Chile - Talca, considerando que un docente universitario debe poseer Dominio del contenido que enseña. Porcentaje alto en comparación de estudios similares, en los que bordea solo en el 14\% (VENTURA et al., 2011) y 4,9\% (CASERO, 2010).

En primera instancia, y lo que inmediatamente nos damos cuenta es el manejo de contenido del docente y desde ahí comienza nuestra evaluación si el docente presenta el correcto uso y manejo de contenido es apto realizar una clase, ya que no puede realizar aprendizaje en los alumnos en forma errónea. (<Elementos internos $\mid 26>-\S 2$ referencias codificadas, Referencia $1^{2}$ ).

Otro punto súper importante es el dominio de contenido que el docente posea, ya sea de su disciplina y además sus contenidos curriculares, es decir que su clase se encuentre bien planificada y no improvisada y que realmente esté basada en lograr aprendizaje. (<Elementos internos $\backslash 19>-\S 3$ referencias codificadas, Referencia 1).

Esta característica pedagógica genera en los estudiantes confianza y seguridad para enfrentarse positivamente al aprendizaje, además, promueve por medio de esta confianza, relaciones positivas que fortalecen la relación profesor-estudiante. Esto produce que el proceso de enseñanzaaprendizaje implementado por el docente genere mayor credibilidad y significado en los estudiantes. Conocer algo nos permite enseñarlo; y conocer un contenido con profundidad significa estar mentalmente organizado y bien preparado para enseñarlo de una forma general, cuestión ampliamente investigativa por los autores que trabajan la noción de conocimiento pedagógico del contenido (AMADE-ESCOT, 2000; BARNETT; HODSON, 2001; FEIMAN-NEMSER, 2001; FENSTERMACHER, 1994).

Para complementar las concepciones de los estudiantes, algunos autores mencionan su punto de vista de acuerdo a lo que significa que el docente domine los contenidos que enseña. El conocimiento pedagógico del contenido se refiere a la habilidad para traducir los contenidos temáticos a un grupo diverso de estudiantes, utilizando estrategias y métodos de instrucción y evaluación múltiple. Teniendo en cuenta las limitaciones contextuales, culturales y sociales en el ambiente de aprendizaje.

2- Códigos de referencia de las entrevistas citadas aquí proporcionados por software Nvivo. 
1.1 Categoría Previa Características Pedagógicas - Categoría Primaria Perfil Pedagógico - Categoría Secundaria: Calidad de la Metodología de Enseñanza

Para un 10,2 \% de los estudiantes a los que se le aplicó el cuestionario, pertenecientes a la Facultad de Educación de la Universidad Autónoma de Chile, sede Talca, la Calidad de la metodología de enseñanza es una de las características más importantes que debe manejar un buen docente universitario. En el estudio realizado en Portugal, los resultados muestran una representatividad menor a la demostrada por los estudiantes de la Facultad de Educación de la Universidad Autónoma, señalando con un 1,5\% de representatividad esta característica (VENTURA et al., 2011) . Teniendo en cuenta los resultados del estudio portugués, podemos verificar que los estudiantes investigados de la Universidad Autónoma de Chile, consideran la Calidad de las metodologías de enseñanza como una práctica docente fundamental para que los alumnos aprendan los contenidos que enseña.

[...] principalmente me centro en la metodología de trabajo del docente, en como plantea el proceso de aprendizaje, que estrategias educativas va utilizando para poder enseñar [...] $\quad(<$ Elementos internos $\backslash 19>-\S 1$ referencia codificada).

En el siguiente relato se señala que el buen docente universitario tiene:

[...] manejo de la materia y de los materiales que nos entregan, pero más que nada es como aborda los temas a tratar, ya que si el profesor no entiende lo que enseña, es imposible que los alumnos aprendan [...] (<Elementos internos\25>- § 1 referencia codificada).

Biggs (2004) señala que uno de los factores más importantes y a la vez más básico para la implementación metodológica del docente, es tener una base de conocimientos bien estructurados, claros y encarnados, de tal forma que el docente sea capaz de formular estrategias para poder entregar ese contenido de forma eficaz, buscando métodos que generen experiencias positivas entorno al aprendizaje ). Las buenas metodologías logran que los estudiantes se interesen por los contenidos entregados, provocando interés en los educandos de tal forma, que potencie instancias de investigación y re-formulación del contenido, tal como lo señala en sus diversos estudios (GARRITZ; TRINIDAD-VELASCO, 2004; GAUTHIER; DE LIMA, 1998).

\subsection{Categoría Previa Características Pedagógicas - Categoría Primaria Perfil Pedagógico - Categoría Secundaria: Metodología de enseñanza comprensible}

El 7,1 \% del total de los estudiantes investigados de la Facultad de Educación de la Universidad Autónoma de Chile, sede Talca, considera que el docente universitario debe implementar en su accionar pedagógico Metodologías de enseñanzas comprensibles. Investigaciones similares dan cuenta una diferencia en la valoración de esta característica, indicando un $2 \%$ de relevancia a esta categoría.

Siendo una de las categorías más relevantes según la percepción de los estudiantes de esta casa de estudios para considerar un buen docente universitario. Shulman (1987) en relación a esta categoría, describe los métodos más útiles de representaciones de estas ideas; las analogías, ilustraciones, ejemplos, explicaciones y demostraciones más significativas. En definitiva, las formas de representación y formulación del contenido que lo hace comprensible a los estudiantes.

\section{Categoría Previa Características Humanas - Categoría Primaria: Características Sociales-Categoría Secundaria: Rasgos interpersonales del buen docente universitario}

Los estudiantes de la Facultad de Educación de la Universidad Autónoma de Chile, sede Talca, consideran relevante esta categoría, 
representando el 34,7 \% de la totalidad de relatos analizados. Los conceptos que componen esta categoría son: profesor apoyador, confiable, con disponibilidad, sociable, integrador, empático, colaborativo, comprensivo, con disposición, comunicativo, interactivo, dialogante, cálido, afectuoso, simpático y serio.

Todo proceso de enseñanza está compuesto por la relación compleja de un docente y sus estudiantes, por lo mismo, el docente debe poseer habilidades sociales y ocuparlas en favor de lograr mejores resultados de aprendizaje, favoreciendo un clima de aula acogedor que invite al trabajo colaborativo entre pares. Algunas referencias de los estudiantes que nos permiten ahondar en el análisis, son las siguientes:

[...] demostrar confianza [...] (<Elementos internos|Facultad de Educación|Pedagogía en ingles $\backslash 9>$ ).

[...] que tengan disponibilidad del tiempo para responder a dudas en clase [...] $\quad(<$ Elementos internos|Facultad de Educación $\mid$ Pedagogía en castellano $\backslash 5>$ ).

[...] un buen docente ha de ser empático [...] $\quad<$ Elementos internos|Facultad de Educación|Pedagogía en artes visuales $\mid 18.341 .995-1>$ ).

Que el profesor sea una persona confiable o empática, parecen ser cualidades que se repiten constantemente entre los estudiantes, otorgándole gran relevancia a los conceptos, por sobre otros de carácter más instrumental. Un profesor que entienda las características de cada alumno parece ser fundamental a la hora de valorar su función en la universidad, entender que cada estudiante es diferente, es decir con un mundo distinto al de los demás, con rasgos físicos, psíquicos, emocionales diferentes al universo de seres humanos, se hace imprescindible al momento de calificar las conductas que se presentan en las clases a impartir. En definitiva, lo que se plantea es que el profesor comprenda que la acción de enseñar es un ejercicio preferentemente de humanización (FREIRE, 1993; MATURANA; PÖRKSEN, 2004).

\subsection{Categoría Previa Características Humanas - Categoría Primaria: Características Sociales-Categoría Secundaria Responsabilidad}

Esta categoría se encuentra inserta dentro de las características humanas que debe poseer un buen docente y que fueron mencionadas en la matriz de sistematización. Esta característica actitudinal mencionada en los relatos presenta una representatividad de 16\% de la categoría previa. En relación a estudios similares, se destaca el trabajo realizado en México donde la responsabilidad es jerarquizada como la característica principal de un buen docente y en el estudio de Ventura et al. (2011) solo representa un 0,8\%. Estas diferencias considerables pueden deberse a la diversidad de instrumentos utilizados para recoger la información.

Los estudiantes de la Facultad de Educación señalan:

[...] responsabilidad ante cada aspecto [...] <Elementos internos|Facultad de Educación|Pedagogía en educación física| ALUMNO $44(\mathrm{M})>$ ).

[...] que demuestre con acciones su discurso, responsable y comprometido [...] $\quad(<$ Elementos internos|Facultad de Educación|Pedagogía en educación física| ALUMNO $40(\mathrm{M})>$ ).

[...] puntual con los horarios, cumplimiento del programa, entrega de evaluaciones [...] $\quad<$ Elementos internos|Facultad de Educación|Pedagogía en educación matemática|ALUMNO $9(\mathrm{M})>$ ).

La responsabilidad junto con características valóricas se transforma en un importante medio calificativo del accionar docente, por sobre aspectos pedagógicos $\mathrm{y}$ académicos. El compromiso es una propiedad fundamental para definir a un buen docente 
universitario, el cual es indispensable para que un docente accione de manera responsable.

Es importante demostrar con ejemplos $\mathrm{y}$ acciones concretas el discurso del profesor, especialmente cuando se exige responsabilidad y compromiso. El docente responsable es aquel que actúa conscientemente siendo él la causa directa o indirecta de un hecho ocurrido. También es el que cumple con sus obligaciones o que pone cuidado y atención en lo que hace o decide.

\section{Categoría Previa Características Ideológicas -} Categoría Primaria: Características de personalidadCaracterísticas secundaria: Rasgos FormativoPedagógico

La categoría secundaria Rasgos formativos pedagógicos obtuvo un 22,5\% de la totalidad de los relatos. Dentro de esta categoría, podemos definir las características asociadas al perfil del docente que despliega en los procesos de enseñanza-aprendizaje. Las más destacadas son: imaginativo, creativo, decidido, flexible, improvisador, lúdico, pionero, inteligente, versátil, entrega, observador, critico, criterioso, coherente, perseverante, actualizado, estratégico, estructurado, exigente, proactivo, reflexivo, con vocación, dedicado, riguroso, sabio, ordenado, culto, competitivo, interesado, contextualizado, informador y autocrítico, atributos que quedan plasmados en las siguientes palabras, cuando por ejemplo los estudiantes aluden a un profesor creativo:

[...] capacidad creativa en cuanto a las actividades de aprendizaje [...] (<Elementos internos|Facultad de Educación|Pedagogía en historia y ciencias sociales $\backslash 1 \mathrm{~F}>$ ).

[...] creatividad frente a los recursos que utiliza [...] $\quad(<$ Elementos internos|Facultad de Educación|Pedagogía en historia $\mathrm{y}$ ciencias sociales $\backslash 15 \mathrm{M}>$ ).

Los relatos destacan la innovación y la creatividad de los docentes en sus prácticas metodológicas, principalmente, por la motivación que esto genera. Incorporar la tecnología o instancias de aprendizaje novedosas para los estudiantes genera una rotura a las prácticas habituales de los docentes, alejándose de los modelos tradicionales y demostrando una capacidad de actualización por parte del docente (DE LA HERRÁN ; PAREDES, 2012).

Otro factor mencionado y con una alta cantidad de referencias, es el carácter lúdico que un buen docente debe plasmar en su accionar, entendiendo el ludismo como la capacidad de otorgarle un sentido grato $\mathrm{y}$ entretenido a las prácticas metodológicas, que muchas veces en la universidad se vuelven monótonas y predecibles. El estudiante exige por tanto una clase llamativa, que junto a una capacidad proactiva y pionera incentive el trabajo colaborativo dentro del aula, lo que queda reflejado en las siguientes referencias de los estudiantes:

[...] que sea lúdico y entretenido [...] (<Elementos internos|Facultad de Educación $\backslash$ Pedagogía en párvulos $\backslash 22>$ ).

[...] tener proactividad para trabajar [...] $\quad<$ Elementos internos|Facultad de Educación|Pedagogía en educación general básica $\mid 16>$ ).

Por último y rescatando las cualidades donde los estudiantes universitarios hacen más referencia, tenemos la capacidad crítica, autocrítico y reflexiva. Características muy difíciles de encontrar puesto que muchas veces el entorno menosprecia y se burla de las opiniones, percepciones y/o sugerencias que puedan surgir, muchas veces ocurre que los mismos estudiantes temen levantar la mano para hablar por el miedo a las burlas o los cuestionamientos poco críticos y reflexivos de sus compañeros e incluso de los docentes. Esos elementos deben primar dentro de las aulas, ya que provocarán un ambiente de crecimiento mutuo en el diálogo y la comunicación favorecerá la creación de nuevas situaciones y/o conocimientos. Tensionar, 
autoanalizarse, criticar con fundamentos, ser consciente de lo que nos ocurre y como reaccionamos, en fin, elementos que un docente debe poseer para que su práctica se mantenga actualizada, contextualizada y genere las estrategias necesarias.

\subsection{Categoría Previa Características Ideológicas - Categoría Primaria: Características de personalidad- Características secundaria: Rasgos humanístico- emocionales del buen docente universitario}

Esta categoría tiene un porcentaje de frecuencia de 18,05\% de la totalidad de referencias analizadas pertenecientes a esta categoría. Cabe destacar que este tipo de características han sido ahondadas en solo un estudio (CASER0, 2010). Cuando hablamos de este tipo de rasgos, es cuando ahondamos en la medula de los sentimientos del profesor y de esto dependerán las reacciones que éste muestre al momento de enfrentar ciertas situaciones. Los estudiantes encuestados apuntan que un buen docente en este aspecto debiese ser alguien con características como; humilde, positivo, espontáneo, energético, animoso, entretenido, seguro, con sentido del humor, valiente, alegre, entusiasta, paciente, sensible, carismático, intuitivo, preocupado, obsesivo y controlado. Estas propiedades nacen de sus palabras, algunas por ejemplo son las siguientes:

[...] que no se crea superior al resto [...] $\quad(<$ Elementos internos|Facultad de Educación|Pedagogía en educación física| ALUMNO 40 (M)>).

[...] ser alegre [...] (<Elementos internos| Facultad de Educación|Pedagogía en educación física \ALUMNO 9 (M)>).

Ocupando estas referencias, cuando hablamos de humildad respondemos a la necesidad de que el docente sea cercano, una persona accesible, con quien se puede dialogar y a quien los estudiantes no temen preguntar, es alguien que no es engreído por su conocimiento, sino que muy por el contrario, es quien quiere compartirlos a sus estudiantes. Esta actitud cercana permite encarar las situaciones diarias de una forma positiva generando ambientes de confianza y alegría entorno al aprendizaje de los estudiantes (DAY, 2006).

\subsection{Categoría Previa Características Ideológicas - Categoría Primaria: Características de personalidad- Características secundaria: Rasgos Ético-Morales de un buen docente universitario}

Respecto de los datos ético-morales recogidos en la investigación, el 11,7\% destacó la relevancia de esta categoría. Dentro de ésta, se destacan los códigos: compromiso con la labor docente, puntualidad, presentación personal y coherencia que debe poseer el docente.

La acción educadora no es simplemente una actividad técnica, que puede repetirse sin reflexión, ni una acción desprovista de comunicación y de contacto social. Exige, por el contrario, una estrecha y confiada relación personal entre el docente y el estudiante, mediado por propiedades éticas y morales que no pueden desarrollarse de forma satisfactoria sin la conciencia social de la acción pedagógica por parte de los agentes educativos. No hay que olvidar que la enseñanza supone una interacción positiva entre un profesor y un grupo de sujetos que asisten a sus clases para formarse como profesionales.

Cuando sehabla deformaciónéticay moral desde la percepción de los sujetos investigados, ellos establecen los siguientes conceptos: honesto, justo, equitativo, higiene personal, presentación personal, valores, correcto, compromiso con el rol docente, compromiso con los estudiantes y consecuentemente, todos estos códigos aluden a las propiedades que un buen docente universitario debe poseer. Los estudiantes encuestados las explicitan con las siguientes palabras:

[...] tener una actitud concreta e igualitaria con todos los alumnos [...] $(<$ Elementos 
internos|Facultad de Educación|Pedagogía en matemáticas $\mid 9>$ ).

[...] presenta una adecuada vestimenta (formal) [...] (<Elementos internos $\mid$ Facultad de Educación|Pedagogía en historia y ciencias sociales $\backslash 8 \mathrm{M}>$ ).

[...] una buena formación valórica [...] $\quad(<$ Elementos internos|Facultad de Educación|Pedagogía en historia y ciencias sociales $\backslash 11 \mathrm{M}>$ ).

[...] el compromiso del docente por cumplir efectivamente su labor como profesor [...] $\quad(<$ Elementos internos|Facultad de Educación $\mid$ Pedagogía en educación general básica $\mid 15>$ ).

Sin embargo, muchas veces se olvida la importancia de estas características en el plano docente; hay que destacar que la ética en los docentes puede ser muy importante, siempre y cuando se logre estimular la reflexión de modo personal y compartida del involucrado sobre las propias actitudes morales como profesional, sobre el contexto que lo rodea y sobre las posibilidades que se le presenten.

Es evidente que la adquisición de los rasgos éticos y morales se da a lo largo de la vida, sin embargo, cuando se decide ser un profesional al servicio de la sociedad, es muy relevante potenciar aún más esta área y para esto se debe retroalimentar no solamente al profesional sino que debe haber una integración de estas características desde la formación del profesor.

Los estudiantes esperan encontrarse con un docente que sea honesto, justo y equitativo, esto quiere decir que de un mismo trato a todos y que tenga la capacidad de reconocer tanto los aciertos como los errores a modo personal y compartido, los estudiantes pretenden que este buen docente demuestre una higiene personal adecuada, desde la perspectiva de su forma de vestir y de su aspecto físico, además de su presentación personal. Relacionado directamente con los valores, se encuentra con la necesidad de contar con un docente correcto que esté comprometido con el rol docente y que esté comprometido con sus estudiantes además de ser consecuente en su actuar y no tenga dobles discursos.

\section{Conclusiones}

En cuanto a la metodología utilizada, el enfoque mixto generó una mayor comprensión del fenómeno investigado en el contexto particular donde el estudio fue llevado a cabo, permitiendo una cobertura de un $80 \%$ del universo estudiado, además de rapidez y profundización de las características más destacadas. Desde esta metodología, fue necesario revisar los datos obtenidos a través de dos análisis, uno de frecuencia y el otro de discurso, otorgando diversidad y riqueza de los análisis.

Los cuestionarios abiertos, otorgaron información relevante para la investigación, permitiendo jerarquizar las características más relevantes que debe poseer un docente universitario. Por medio de la frecuencia obtenida, se procedió a entrevistar a sujetos clave de cada carrera con el fin de profundizar y saturar la información relevante.

En un primer momento de análisis, por medio de cuestionarios, las características más destacadas por los estudiantes de la Facultad de Educación de la Universidad Autónoma de Chile, sede Talca, al momento de valorar a un buen docente universitario trascienden al hecho pedagógico o ámbito disciplinar del curso que enseña. Las características humanas e ideológicas en este estudio presentan un mayor porcentaje de representatividad para los estudiantes que lo pedagógico. Desde esta idea, el buen docente universitario de la Facultad de Educación de la Universidad Autónoma de Chile, sede Talca, es: una persona confiable, sociable, cercana, integradora, empática, colaborativa, comprensiva, segura, paciente, humilde, alegre y respetuosa; todas ellas características cotidianas y académicas que dejan ver cómo el rol de los docentes que forman parte del contexto investigado no se acaba una vez que está fuera de la institución formadora, sino 
que traspasa estos límites y envuelve la acción diaria que el docente realiza.

En un segundo momento de análisis, se constata la necesidad de contar con un docente que posea dominio de contenido; esta característica permite la articulación de conocimientos procedentes de la formación profesional y disciplinar de los docentes, con la experiencia construida a lo largo de su trayectoria profesional. Es el docente en estudio el encargado de construir y movilizar los saberes propios de su experiencia profesional, a través de una reflexión crítica de su hacer y un compromiso ineludible con la acción de educar, resultando finalmente el despliegue de un conocimiento didáctico, metodológico y disciplinar en el aula. A través de esto, el docente transmite seguridad en el estudiante, permitiendo conocimientos que trascienden en su formación.

Finalmente, el buen docente universitario del contexto investigado necesita poseer características que transiten entre las habilidades pedagógicas, humanas e ideológicas, enfatizando en todo momento su accionar en y desde el aprendizaje, a través de un proceso de enseñanza pensado en el estudiante que se está formando.

\section{Referencias}

AMADE-ESCOT, Chantal. The contribution of two research programs on teaching content: "Pedagogical content knowledge" and "Didactics of physical education". Journal of Teaching Physical Education, Champaign, v. 20, n. 1, p. 78-101, 2000.

BARNETT, John; HODSON, Derek. Pedagogical context knowledge: toward a fuller understanding of what good science teachers know. Science Education, Florida, v. 85, n. 4, p. 426-453, 2001.

BIGGS, John. Calidad del aprendizaje universitario. Madrid: Narcea, 2004.

BISQUERRA, Rafael. Métodos de investigación educativa: guía práctica. Barcelona: Ceac, 1989.

BOTELLO, Carlos; BONVALLET, Rodrigo. Identificacion de competencias del docente de excelencia de la Universidad del Bio-Bio. Theoria, Concepción, v. 11, n. 1, p. 77-85, 2002.

BOYD, Donald et al. Teacher preparation and student achievement. Educational Evaluation and Policy Analysis, Washington, v. 31, n. 4, p. 416-440, 2009.

CABALÍN, Daisy et al. Concepción de estudiantes y docentes del buen profesor universitario: Facultad de Medicina de la Universidad de La Frontera. International Journal of Morphology, Temuco, v. 28, n. 1, p. 283-290, 2010.

CALV0, Carlos. La formación del profesor como investigador. Temas de Educación, La Serena, n. 9, p. 7-12, 2002.

CAREAGA, Adriana. El desafío de ser docente. Montevidéu: Universidad de la República, 2007.

CASERO, Antonio. ¿ Cómo es el buen profesor universitario según el alumnado? Revista Española de Pedagogía, Madrid, v. 62, n. 246, p. 223-242, 2010.

CHILE. Ministerio de Educación. Ley no 20.129 del 17 de noviembre de 2006. Sistema Nacional de Aseguramiento de la Calidad de la Educación Superior. Santiago de Chile, Ministerio de Educación de Chile, 2006. Disponible en: <https://www. leychile.cl/Navegar?idNorma=255323\&idVersion=2016-04-01>. Acceso en: jul. 2015.

CHILE. Ministerio de Educación. Educación Superior. Santiago de Chile: Ministerio de Educación de Chile, 2016. Disponible en: $<$ http://www.mineduc.cl/>. Acceso en: jul. 2015.

CORRÊA, Guilherme; RIBEIRO, Victoria. A formação pedagógica no ensino superior e o papel da pós-graduação stricto sensu. Educação e Pesquisa, São Paulo, v. 39, n. 2, p. 319-334, 2013. 
DAVILA, Andrés. Las perspectivas metodológicas cualitativa y cuantitativa en las ciencias sociales: debate teórico e implicaciones praxeológicas. In: DELGADO, José María. Métodos y técnicas cualitativas de investigación en ciencias sociales. Madrid: Síntesis, 1999. p. 69 - 86.

DAY, Christopher. Pasión por enseñar: la identidad personal y profesional del docente y sus valores. Madrid: Narcea, 2006.

DE LA HERRÁN, Agustín; PAREDES, Joaquín. Tecnología y creatividad en la mejora de la docencia universitaria. REICE: Revista Electrónica Iberoamericana sobre Calidad, Eficacia y Cambio en Educación, Madrid, v. 10, n. 2, p. 56-67, 2012.

DELORS, Jacques. La educación encierra un tesoro. Madrid: Santillana: Unesco, 1996.

FEIMAN-NEMSER, Sharon. From preparation to practice: designing a continuum to strengthen and sustain teaching. The Teachers College Record, Columbia, v. 103, n. 6, p. 1013-1055, 2001.

FENSTERMACHER, Gary. The knower and the known: the nature of knowledge in research on teaching. Review of Research in Education, Washington, v. 20, p. 3-56, 1994.

FERNÁNDEZ, Manuela; GONZÁLEZ, Sebastián. El perfil del buen docente universitario: una aproximación en función del sexo del alumnado. REDU: Revista de Docencia Universitaria, Valencia, v. 10, n. 2, p. 237-249, 2012.

FREIRE, Paulo. La educación como práctica de la libertad. Buenos Aires: Siglo XXI, 1978.

FREIRE, Paulo. Pedagogía de la esperanza: un reencuentro con la pedagogía del oprimido. Buenos Aires: Siglo XXI, 1993.

GARCÍA, Maria del Mar; DOMINGO, Begoña; DíAZ, José. El perfil del profesor universitario de calidad desde la perspectiva del alumnado. Educación XXI, Madrid, v. 9, n. 1, p. 183-198, 2006.

GARRITZ, Andoni; TRINIDAD-VELASCO, Rufino. El conocimiento pedagógico del contenido. Educación Química, Ciudad de México, v. 15, n. 2, p. 98-102, 2004.

GAUTHIER, Clemont; DE LIMA, Francisco Pereira. Por uma teoria da pedagogia: pesquisas contemporâneas sobre 0 saber docente. ljuí: Unijuí, 1998.

HARRIS, Douglas; SASS, Tim. Teacher training, teacher quality and student achievement. Journal of Public Economics, London, v. 95, n. 7, p. $798-812,2011$

HIRSCH, Ana; PÉREZ, Judith. Rasgos de ser un buen profesional en los alumnos de posgrado. Los casos de la Universidad de Valencia y de la Universidad Nacional Autónoma de México. Educación, Valores y Desarrollo Moral, Ciudad de México, v. 1, p. 73-102, 2006.

LÓPEZ, Bernardo et al. Estilos docentes de los profesores universitarios: la percepción de los alumnos de los buenos profesores. Revista Iberoamericana de Educación, Madrid, n. 51/4, p. 1-16, 2010.

LOPÉZ DE MATURANA, Silvia. Maestros en el territorio, 2012. (Monografia) - Universidad La Serena, La Serena, 2012.

LUNA-SERRANO, Edna; VALLLE-ESPINOSA, María del Consuelo; OSUNA-LEVER, Cecilia. Los rasgos de un" buen profesional", según la opinión de estudiantes universitarios en México. Revista Electrónica de Investigación Educativa, Baja California, v. 12, n. Spe, p. 1-14, 2010.

MATURANA, Humberto; PÖRKSEN, Bernhard. Del ser al hacer. Los orígenes de la biología del conocer. Revue Européenne de Systémique (Res-Systematica), París, v. 197, n. 2, p. 815-816, 2004.

MUNDINA, Julia; POMBO, María; RUIZ, Elena. La docencia y la tutoría en el nuevo marco universitario. Revista Electrónica Interuniversitaria de Formación del Profesorado, Zaragoza, v. 8, n. 5, p. 12, 2005.

MURCIA, Napoleón; JARAMILLO, Luis Guillermo. La complementariedad etnográfica. Investigación cualitativa: una guía posible para abordar estudios sociales. Armenia: Kinesis, 2000. 
RICE, Jennifer. Teacher quality: understanding the effectiveness of teacher attributes. Washington: ERIC, 2003.

RICOY, Carmen. Contribución sobre los paradigmas de investigación. Educação, Santa Maria, v. 31, n. 1, p. 11-22, 2006.

SANTOS GUERRA, Miguel Angel. Hacer visible lo cotidiano: teoría y práctica de la evaluación cualitativa de los centros escolares. Madrid: Akal, 1998.

SCHÖN, Donald. Educando o profissional reflexivo. Porto Alegre: Penso, 2010.

SHULMAN, Lee. Knowledge and teaching: foundations of the new reform. Harvard Educational Review, Cambridge, v. 57, n. 1, p. 1-23, 1987.

SHULMAN, Lee. Those who understand: knowledge growth in teaching. Educational Researcher, Tallahassee, v. 15, n. 2, p. 4-14, 1986.

SIMONS, Helen. El estudio de caso: teoría y práctica. Madrid: Morata, 2011.

TARDIF, Maurice. Los saberes del docente y su desarrollo profesional. Madrid: Narcea, 2004.

VENTURA, Maria et al. 0 bom professor: opinião dos estudantes. Revista de Enfermagem Referência, Coimbra, série 3, n. 5, p. 95-102, 2011.

Recibido en: 03.08.2015

Aprobado en: 12.04.2016

Eugenio Merellano-Navarro es doctorando en la Universidad de Extremadura, magíster en Motricidad Humana mención Motricidad Educativa, Universidad Autónoma de Chile. Docente asistente de la Universidad Autónoma de Chile, sede Talca.

Alejandro Almonacid-Fierro es doctorando Universidad de Extremadura, magíster en Motricidad Humana mención Motricidad Educativa, Universidad Autónoma de Chile. Docente asociado de la Universidad Autónoma de Chile, sede Talca.

Alberto Moreno-Doña es doctor por la Universidad de Granada, magíster en Estudios Latinoamericanos de la Universidad de La Serena, Chile. Profesor adjunto en la Escuela de Educación Parvularia de la Universidad de Valparaíso, Chile.

Cesar Castro-Jaque es magíster en Motricidad Humana mención Motricidad Educativa, Universidad Autónoma de Chile. Profesor de Educación Física de la Pontificia Universidad Católica de Chile. Docente asistente de la Universidad Autónoma de Chile, sede Talca. 